\title{
Development of a Mouse Model of Ischemic Osteonecrosis
}

\author{
Nobuhiro Kamiya MD, PhD, Ryosuke Yamaguchi MD, PhD, \\ Olumide Aruwajoye MS, Naga Suresh Adapala PhD, \\ Harry K. W. Kim MD, MS
}

Received: 1 July 2014 / Accepted: 27 January 2015 / Published online: 10 February 2015

(C) The Association of Bone and Joint Surgeons (B) 2015

\begin{abstract}
Background Availability of a reliable mouse model of ischemic osteonecrosis could accelerate the development of novel therapeutic strategies to stimulate bone healing after ischemic osteonecrosis; however, no mouse model of ischemic osteonecrosis is currently available.

Questions/purposes To develop a surgical mouse model of ischemic osteonecrosis, we asked, (1) if the blood vessels that contribute to the blood supply of the distal femoral epiphysis are cauterized, can we generate an osteonecrosis mouse model; (2) what are the histologic changes observed
\end{abstract}

The institution of one or more of the authors has received, during the study period, funding from the Pediatric Orthopaedic Society of North America (HKWK), and an intramural grant from Texas Scottish Rite Hospital for Children (NK and HKWK).

All ICMJE Conflict of Interest Forms for authors and Clinical Orthopaedics and Related Research editors and board members are on file with the publication and can be viewed on request.

Each author certifies that his or her institution approved the animal protocol for this investigation and that all investigations were conducted in conformity with ethical principles of research.

This study was performed at Texas Scottish Rite Hospital for Children and University of Texas Southwestern Medical Center, Dallas, TX, USA.

N. Kamiya, R. Yamaguchi, O. Aruwajoye,

N. S. Adapala, H. K. W. Kim ( $\square)$

Center of Excellence in Hip Disorders, Texas Scottish Rite

Hospital for Children, 2222 Welborn Street, Dallas, TX 75219,

USA

e-mail: Harry.Kim@tsrh.org

N. Kamiya, R. Yamaguchi, N. S. Adapala, H. K. W. Kim Department of Orthopaedic Surgery, University of Texas Southwestern Medical Center, Dallas, TX, USA

N. Kamiya

Sports Medicine, Tenri University, Tenri, Nara, Japan in this mouse model, and (3) what are the morphologic changes in the model.

Methods We performed microangiography to identify blood vessels supplying the distal femoral epiphysis in mice, and four vessels were cauterized using microsurgical techniques to induce ischemic osteonecrosis. Histologic assessment of cell death in the trabecular bone was performed using terminal deoxynucleotidyl transferase mediated dUTP nick-end labeling (TUNEL) and counting the empty lacunae in three serial sections. Quantitation of osteoclast and osteoblast numbers was performed using image analysis software. Morphologic assessments of the distal femoral epiphysis for deformity and for trabecular bone parameters were performed using micro-CT.

Results We identified four blood vessels about the knee that had to be cauterized to induce total ischemic osteonecrosis of the distal femoral epiphysis. Qualitative assessment of histologic sections of the epiphysis showed a loss of nuclear staining of marrow cells, disorganized marrow structure, and necrotic blood vessels at 1 week. By 2 weeks, vascular tissue invasion of the necrotic marrow space was observed with a progressive increase in infiltration of the necrotic marrow space with the vascular tissue at 4 and 6 weeks. TUNEL staining showed extensive cell death in the marrow and trabecular bone 24 hours after the induction of ischemia. The mean percent of TUNELpositive osteocytes in the trabecular bone increased from $2 \% \pm 1 \%$ in the control group to a peak of $98 \% \pm 3 \%$ in the ischemic group 1 week after induction of ischemia (mean difference, 96\%; 95\% CI, 81\%-111\%; $\mathrm{p}<0.0001$ ). The mean percent of empty lacunae increased from $1 \% \pm 1 \%$ in the control group to a peak of $78 \% \pm 15 \%$ in the ischemic group at 4 weeks (mean difference, $77 \%$; $95 \%$ CI, 56\%-97\%; p < 0.0001). Quantitative analysis showed that the mean number of osteoclasts per bone surface was 
decreased in the ischemic group at 1,2 , and 4 weeks ( $\mathrm{p}<0.0001,<0.0001$, and $\mathrm{p}=0.02$, respectively) compared with the control group. The mean number of osteoclasts increased to a level similar to that of the control group at 6 weeks $(p=0.23)$. The numbers of osteoblasts per bone surface were decreased in the ischemic group at 1 , 2 and 4 weeks $(p<0.0001$ for each) compared with the numbers in the control group. The mean number of osteoblasts also increased to a level similar to that of the control group at 6 weeks $(p=0.91)$. Mean bone volume percent assessed by micro-CT was lower in the ischemic group compared with the control group from 2 to 6 weeks. The mean differences in the percent bone volume between the control and ischemic groups at 2, 4, and 6 weeks were $5.5 \%$ (95\% CI, $0.9 \%-10.2 \% ; \mathrm{p}=0.01), 5.3 \%$ (95\% CI, $0.6 \%-9.9 \% ; \mathrm{p}=0.02)$, and $6.0 \%(95 \% \mathrm{CI}, 1.1 \%-10.9 \%$; $\mathrm{p}=0.008$ ), respectively. A deformity of the distal femoral epiphysis was observed at 6 weeks with the mean epiphyseal height to width ratio of $0.74 \pm 0.03$ in the control group compared with $0.66 \pm 0.06$ in the ischemic group (mean difference, $0.08 ; 95 \% \mathrm{CI}, 0.00-0.16 ; \mathrm{p}=0.03$ ).

Conclusion We developed a novel mouse model of ischemic osteonecrosis that produced extensive cell death in the distal femoral epiphysis which developed a deformity with time.

Clinical Relevance The new mouse model may be a useful tool to test potential therapeutic strategies to improve bone healing after ischemic osteonecrosis.

\section{Introduction}

Traumatic injuries leading to fracture, fracture-dislocation, or dislocation can acutely disrupt the blood supply to the bone, producing ischemic osteonecrosis. Femoral neck fracture or hip dislocation, proximal humeral fracture (or fracture-dislocation), talar neck fracture, scaphoid fracture, and lateral condyle facture are some of the injuries that may disrupt the blood supply, and in susceptible bones, produce ischemic osteonecrosis [1, 30, 39, 42, 48]. Advances in internal fixation of these fractures have been made; however, no similar advances have been made for treatment of osteonecrotic bone.

Various animal models of osteonecrosis are available to study the pathophysiology and treatment of osteonecrosis. These models can be categorized broadly as traumatic and nontraumatic osteonecrosis [2, 3, 11], such as corticosteroid [17, 19, 46, 47], lipopolysaccharide [16], alcohol [45], cryogen [20, 40], and immune reaction-induced models of osteonecrosis $[32,36]$. The site of osteonecrosis in these models, however, is limited to the femoral head except in corticosteroid-induced models which tend to develop multifocal lesions. The experimental models also can be divided into large animal models such as canines [31, 34, 37], porcines [24, 41], and sheep [44], and small animal models such as rabbits $[5,15-17,19-21]$ and rats $[14,29,38]$. These models can be further classified into immature and mature animal models that represent pediatric and adult osteonecrotic conditions [11].

Although there are many experimental models of osteonecrosis, we recognized a need to develop a mouse model of ischemic osteonecrosis to investigate the molecular mechanisms involved in the repair processes after ischemic osteonecrosis, such as angiogenesis, osteoclastogenesis, and osteogenesis. In vivo studies using genetically engineered mouse strains are invaluable for this purpose, as seen in the field of fracture repair where a specific gene in the molecular signaling pathway can be deleted or made to be overexpressed $[10,12,43]$. The loss of function and gain of function experiments using genetically engineered mouse strains have led to new insights in growth factors [12, 13, 27, 43] and transcription factors that control fracture healing $[6,10]$. Currently, there is no mouse model of ischemic osteonecrosis produced by disruption of the blood supply to the bone. Unavailability of such a model is an obstacle to using genetically engineered mouse strains to better understand the molecular pathways involved with the repair process. Development of a mouse model of ischemic osteonecrosis has several other advantages such as lower animal and housing costs, availability of commercial reagents, assays, and analytic kits made specifically for use with mice, and availability of drugs including monoclonal antibodies that have been tested on mice. These are practical considerations which are likely to accelerate the development and testing of novel therapeutic strategies to stimulate bone healing after ischemic osteonecrosis.

The purpose of this study therefore was to develop a surgical model of ischemic osteonecrosis in mice. We asked (1) if the blood vessels that contribute to the blood supply to the distal femoral epiphysis are cauterized, can we generate an osteonecrosis mouse model, (2) what are the histologic changes in the mouse model, and (3) what are the morphologic changes in the model.

\section{Materials and Methods}

Mice

The animal protocols for this study were approved by the Institutional Animal Care and Use Committee of the University of Texas Southwestern Medical Center. Fiveweek-old $\mathrm{C} 57 \mathrm{bl} / 6$ male mice were used in the study (Table 1). 
Table 1. Numbers of mice used in the different studies

\begin{tabular}{ll}
\hline Study & Number of mice \\
\hline $\begin{array}{l}\text { 1. Define vascular anatomy of distal femoral epiphysis using microangiography } \\
\text { 2. Cauterization of different vessels four groups: sham, branch of popliteal vessel only, } \\
\text { popliteal vessel + central genicular vessel, and all four vessels }\end{array}$ & 6 \\
3. Reliability of four-vessel cauterization on producing a total epiphyseal necrosis & 5 mice/group \\
4. Histologic and morphologic analyses & 15 \\
Sham groups: $1,2,4,6$ weeks & 60 \\
Postischemia groups: $1,2,4,6$ weeks & 5 mice/group \\
\hline
\end{tabular}

\section{Microangiography for Vessel Identification}

To define the vascular anatomy around the knee, the microangiographic technique described by Duvall et al. [9] was used in a pilot study of six mice. After euthanization, the thoracic cavity of each mouse was opened and the inferior vena cava was severed to drain the blood. The vasculature was flushed with $0.9 \%$ normal saline containing heparin sodium $(100 \mathrm{U} / \mathrm{mL})$, at an approximate pressure of $100 \mathrm{~mm} \mathrm{Hg}$ via a needle inserted in the left ventricle. The specimens then were pressure-fixed with $10 \%$ neutral buffered formalin. The formalin solution was flushed from the vessels using heparinized saline and the vasculature was injected with a radiopaque silicone rubber compound containing lead chromate (MICROFIL ${ }^{\circledR}$ MV122; Flow Tech Inc, Carver, MA, USA). The microangiography was performed using micro-CT ( $\mu \mathrm{CT} 40$; Scanco Medical; Bassersdorf, Switzerland) to observe the blood vessels and the bone (8-36 $\mu$ isotropic voxel size, $55 \mathrm{kVp}$, and current of $145 \mathrm{~mA}$ ). The resolution was set to medium, which created a $1024 \times 1024$ pixel image matrix. Serial tomograms were reconstructed from the raw data using a cone-beam filtered back-projection algorithm. By defining the vasculature around the knee (Fig. 1), we were able to identify the four vessel groups supplying the distal femoral epiphysis: a branch of the popliteal vessel; and medial, central, and lateral genicular vessels.

Induction of Ischemic Osteonecrosis in the Distal Femoral Epiphysis

Five-week-old C57BL/6 male mice were anesthetized with isoflurane. The blood vessels supplying the right distal femoral epiphysis (ie, a branch of a popliteal and branches of medial, central, lateral genicular vessels) were identified and cauterized using a dissection microscope with $\times 6$ to $\times 40$ objectives and microsurgical instruments to induce ischemic osteonecrosis. The four blood vessels were first cauterized either individually or in combinations of two or more vessels to determine the efficiency of ischemia induction. For the popliteal vessel, a posteromedial approach to the back of the knee was used. To observe the medial, central, and lateral genicular vessels, a medial parapatellar incision was used to perform a knee arthrotomy. The incision and arthrotomy were closed with Number 8-0 sutures. The ischemia-induction surgery took approximately 30 minutes for each mouse. In the sham group, the vessels of the right distal femur were exposed as described above but no cauterization was performed. The left distal femoral epiphysis, which received no surgery, was used as the normal control group.

\section{Collection and Processing of Knee Region}

for Histomorphometric Analysis

Mice were sacrificed at designated times after induction of ischemic osteonecrosis ( 1 week, 2 weeks, 4 weeks, and 6 weeks; $n=10$ per time). One animal in the 6 -week group died and was not replaced. The mice in the shamoperated group were sacrificed at 1 to 6 weeks after surgery $(\mathrm{n}=5$ per time). Immediately after sacrifice, the distal femur and proximal tibia of the untreated side (left) and the ischemic side (right) were harvested and fixed with $10 \%$ formalin for 5 days followed by micro-CT, as described below. After scanning, the specimens were decalcified with $10 \%$ EDTA for 5 days. Bones were embedded in paraffin and cut 4 to $5 \mu$ thickness for hematoxylin and eosin, terminal deoxynucleotidyl transferase mediated dUTP nick-end labeling (TUNEL), and tartrate-resistant acid phosphatase (TRAP) staining.

\section{Histomorphometric Analysis}

Hematoxylin and eosin staining was performed following a standard protocol. To detect osteoclasts, TRAP staining was performed as previously reported [23]. To observe cell death in the area of osteonecrosis, TUNEL staining was 

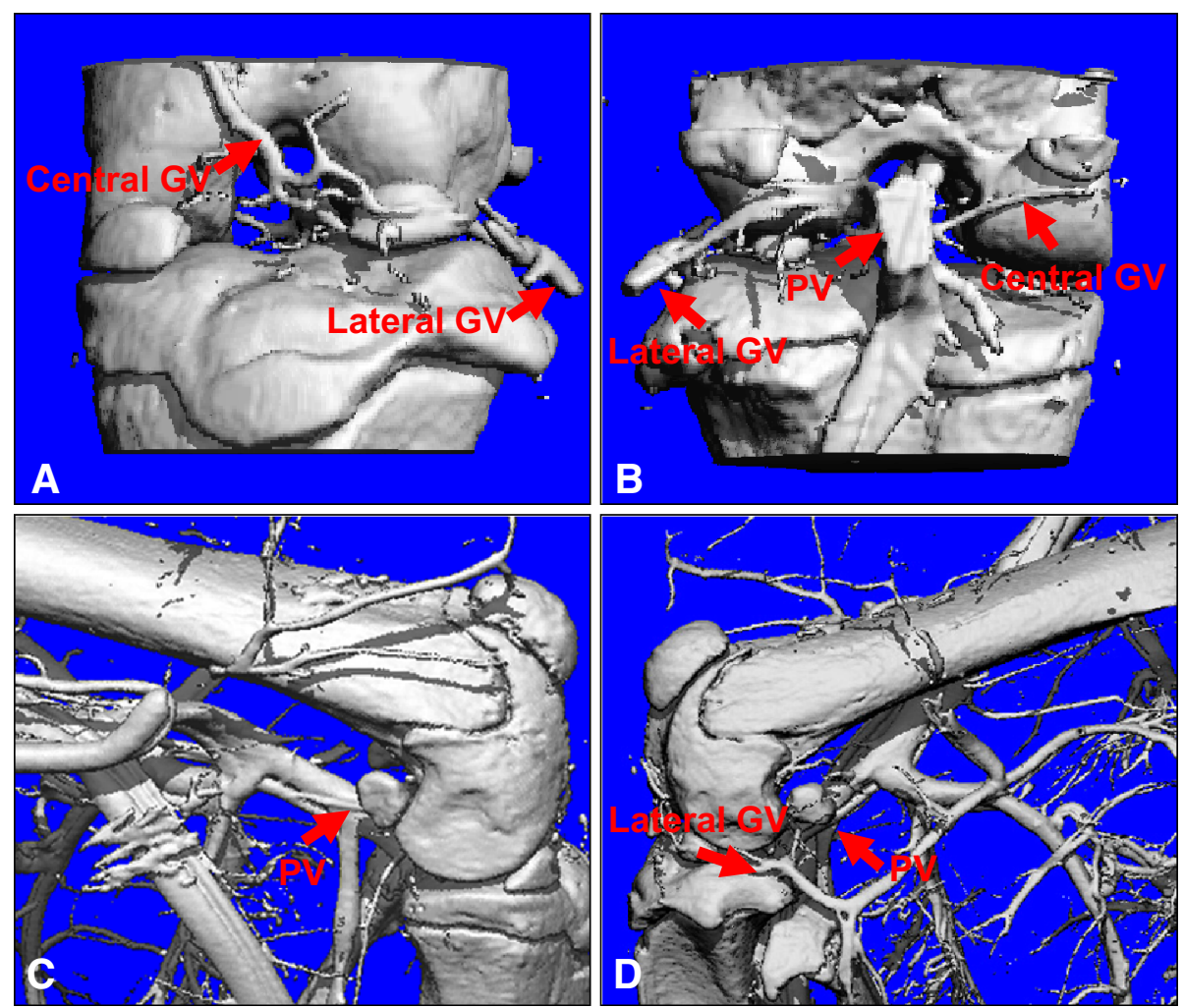

Fig. 1A-D Microangiography was used to define the vascular anatomy around the knee. Vessels were observed by perfusion of the lower limb vasculature with MICROFIL ${ }^{\circledR}$ and micro-CT. The (A) anterior and (B) posterior views of the popliteal fossa, and (C) medial and (D) lateral views of the knee are shown in these three-

performed using an In Situ Cell Death Detection Kit (Roche Applied Science; Roche, Basel, Switzerland) as previously reported [28].

Histologic sections from distal femoral epiphyses were examined using the BIOQUANT ${ }^{\circledR}$ OSTEOIMAGER (BIOQUANT Image Analysis Corp, Nashville, TN, USA) to determine the trabecular bone surface length, number of osteoclasts, number of osteoblasts, number of empty lacunae, number of total trabecular lacunae, number of TUNEL-positive osteocytes, and total number of osteocytes. For quantitative analysis (eight mice/group for all groups except seven for the 6-week group), measurements were performed on three serial sections using BIOQUANT ${ }^{\circledR}$ OSTEO software (version 13.2.60; BIOQUANT Image Analysis Corp) by an observer blinded to the groups. Osteocyte lacunae were considered empty with the absence of cell body in the lacunae or if they only contained pyknotic nucleus [46] in three serial sections. The number of osteoclasts was determined by counting the number of TRAP-positive cells adherent to bone surface and the values were normalized to the bone surface. Osteoblasts were identified as cuboidal cells attached to the bone in regions of bone formation. The percentages of TUNEL- dimensional reconstructed micro-CT images. The arrows indicate vessels supplying the distal femoral epiphysis, which include a branch of the popliteal vessel (PV) and the medial, central, and lateral genicular vessels $(\mathrm{GV})$.

positive osteocytes were obtained by counting and dividing the number of TUNEL-positive osteocytes by the total number of osteocytes in the trabecular bone of the distal femoral epiphysis. The percent empty lacunae was obtained by counting and dividing the number of empty lacunae by the total number of lacunae present in the trabecular bone [8].

\section{Micro-CT Analysis of Distal Femoral Epiphysis}

The distal femoral epiphyses were scanned using SKYSCAN 1172 micro-CT system (Skyscan, Aartselaar, Belgium) with the following settings: $6-\mu$ thickness, $50 \mathrm{kV}$ of energy, and $200 \mathrm{~mA}$ of intensity. Trabecular bone parameters, including bone volume, trabecular thickness, trabecular number, and trabecular separation, were obtained. The following number of specimens was analyzed for each time: 1 week $(n=10), 2$ weeks $(n=10)$, 4 weeks $(\mathrm{n}=10)$, and 6 weeks $(\mathrm{n}=9)$. On each coronal section, the region of interest (ie, trabecular bone in the distal femoral epiphysis) was outlined and the cortical bone surrounding the trabecular bone was excluded. All 

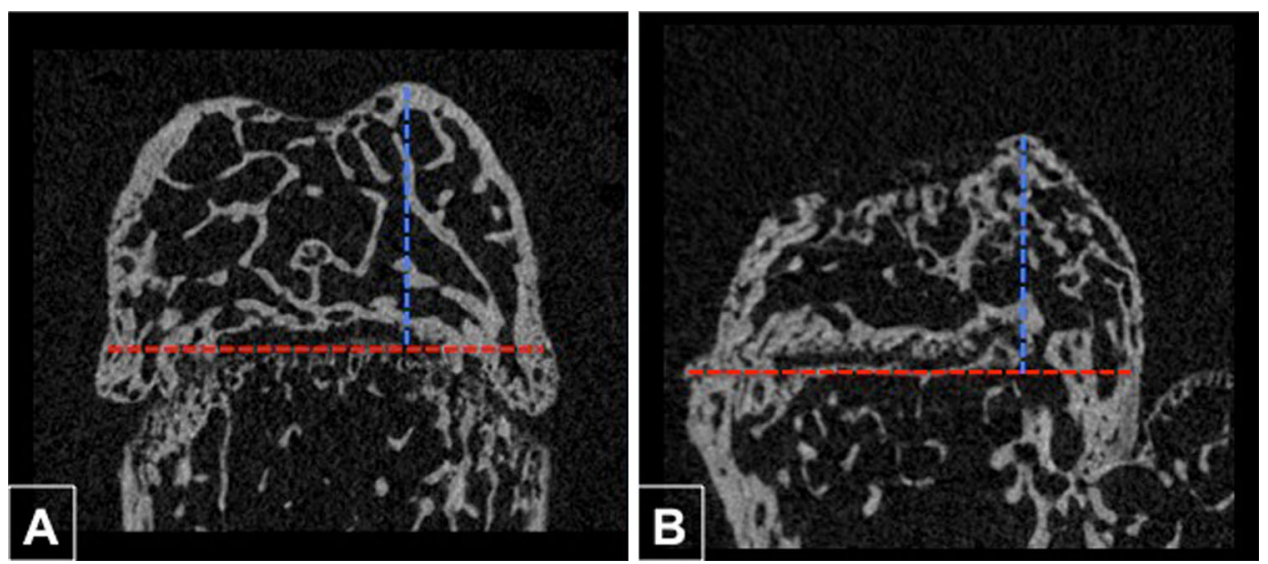

Fig. 2A-B The micro-CT images show mid-coronal region of the distal femoral epiphyses in the (A) control and the (B) ischemic sides 6 weeks after induction of ischemic osteonecrosis. The height (blue

coronal images of the distal femoral epiphysis were analyzed for each specimen, and the mean $\pm \mathrm{SD}$ (standard deviation) value for each trabecular parameter was obtained.

The shape of the distal femoral epiphysis was assessed for deformity and the degree of epiphyseal collapse was evaluated by measuring the height and width of the epiphysis in a mid-coronal plane on micro-CT images (Fig. 2). A ratio of the height and width was calculated for each epiphysis. A lower ratio of height/width would indicate a greater deformity.

\section{Statistical Analysis}

Data are expressed as mean $\pm \mathrm{SD}$. For micro-CT and quantitative histologic analyses, ANOVA and Tukey's multiple comparison tests were used. A p value less than 0.05 was considered statistically significant.

\section{Results}

Vessel Cauterization and Creation of the Mouse Osteonecrosis Model

The cauterization of a branch of the popliteal vessel alone or a branch of the popliteal vessel and a central genicular vessel showed only a partial region of cell death in the distal femoral epiphysis assessed by TUNEL staining (Fig. 3). In contrast, cauterization of all four vessels (popliteal vessel plus all three genicular vessels) produced cell death in the whole distal femoral epiphysis with TUNEL stain-positive cells present throughout the region (Fig. 3). TUNEL stain-positive cells were not increased in the sham-operated group (Fig. 4). The reliability of the line) and width (red line) of the epiphysis were measured in a midcoronal plane. A ratio of the height and width was calculated for each epiphysis.

four-vessel cauterization to produce total epiphyseal osteonecrosis was further assessed using 15 animals. Evidence of extensive cell death in the bone marrow space and the trabecular bone on TUNEL staining was seen in all 15 animals. Based on these findings, we concluded that cauterization of all four vessels was required to induce severe ischemic osteonecrosis in the distal femoral epiphysis. We thus cauterized all four vessels as our standard procedure during the rest of this study.

\section{Histologic Changes in the Model}

Cauterization of all four vessels supplying the distal femoral epiphysis resulted in extensive cell death followed by revascularization, bone resorption, and new bone formation during the 6-week time. Qualitative assessment of histologic sections of the epiphysis (Fig. 5A) showed a loss of nuclear staining of marrow cells, disorganized marrow structure, and necrotic blood vessels at 1 week. By 2 weeks, vascular tissue invasion of the necrotic marrow space was observed, with a progressive increase in infiltration of the necrotic marrow space with vascular tissue at 4 and 6 weeks. In some areas of the epiphysis, hematopoietic marrow was present at 6 weeks. Shamoperated mice showed no evidence of osteonecrosis.

Assessment of the trabecular bone for osteocyte death using TUNEL staining showed an increase in the number of TUNEL-positive osteocytes expressed as a percentage of the total number of osteocytes in the ischemic group at 1,2, and 4 weeks (Fig. 5B). The mean percent of TUNELpositive osteocytes in the trabecular bone was $2 \% \pm 1 \%$ in the control group compared with $98 \% \pm 3 \%$ in the ischemic group at 1 week (mean difference, $96 \%$; 95\% CI, $81 \%-111 \% ; \mathrm{p}<0.0001$ ), $92 \% \pm 7 \%$ at 2 weeks (mean 

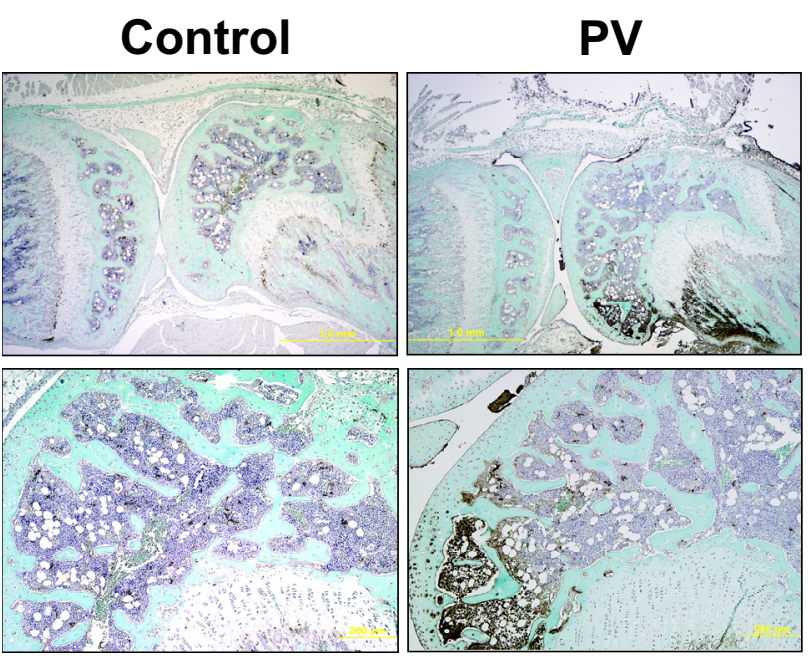

Fig. 3 Development of ischemic osteonecrosis of the distal femoral epiphysis was assessed by TUNEL staining 24 hours postischemia surgery. Cell death in the distal femur was assessed using TUNEL staining. Brown staining shows TUNEL-positive cells. Different combinations of the vessels (ie, a branch of the popliteal vessel [PV] and lateral, central, and medial genicular vessels [GV]) were cauterized and the extent of cell death in the distal femoral epiphysis was compared ( $\mathrm{n}=5 \mathrm{mice} / \mathrm{group}$ ). Cauterization of a branch of the
PV+Central GV

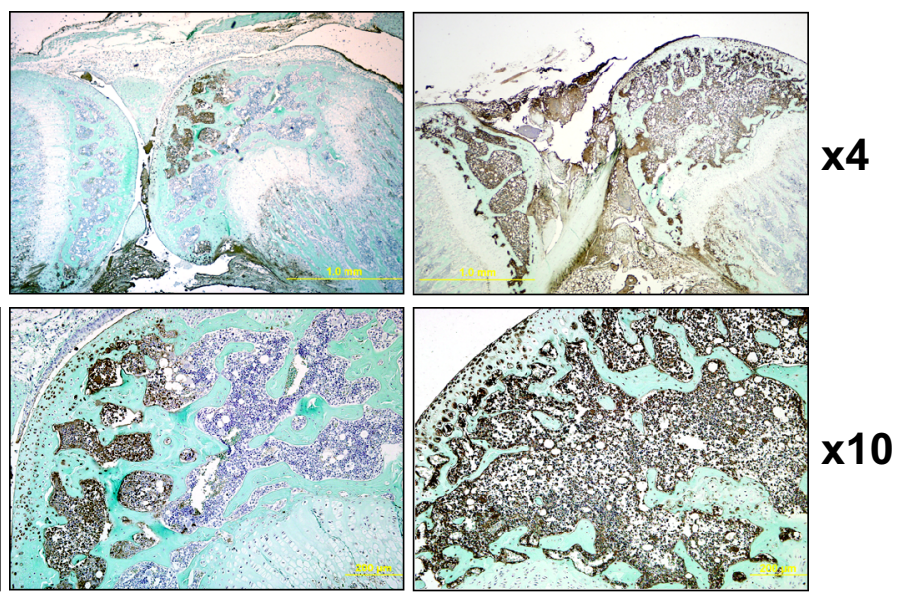

popliteal vessel or a branch of the popliteal vessel plus central genicular vessel produced partial necrosis of the distal femoral epiphysis, as evidenced by partial TUNEL staining in the epiphysis. In contrast, cauterization of all four vessels produced total necrosis of the epiphysis. The control was the untreated left femoral epiphysis (Stain, hematoxylin \& eosin and fast green counterstains; original magnification, $1 \mathrm{~mm} ;=\times 4,50 \mu=\times 10$ ).

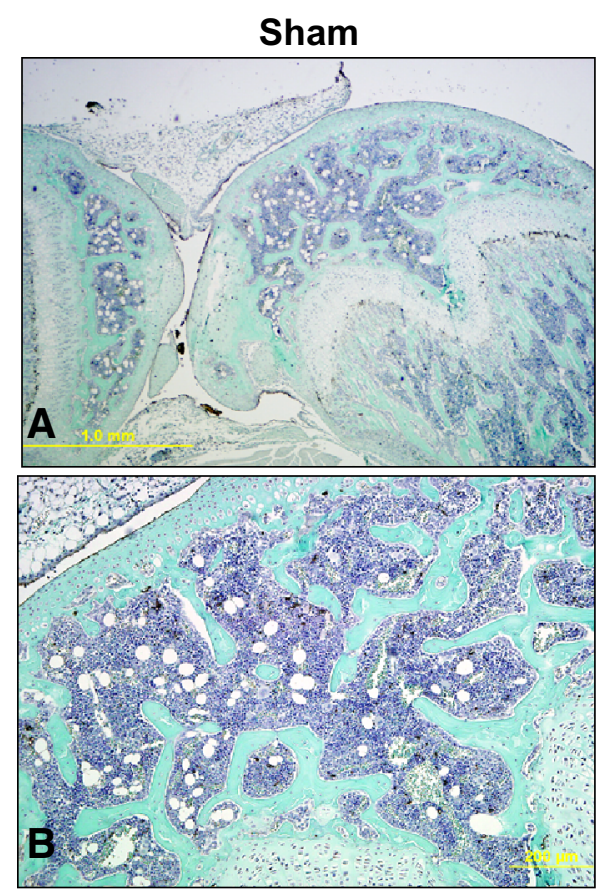

Fig. 4A-D Development of ischemic osteonecrosis of the distal femoral epiphysis was assessed by TUNEL staining 24 hours postischemia surgery. TUNEL staining was not observed in the sham group at either $(\mathbf{A}) \times 4$ or $(\mathbf{B}) \times 10$ magnification. The negative

difference, 90\%; 95\% CI, 74\%-105\%; p < 0.0001), and $84 \% \pm 19 \%$ at 4 weeks (mean difference, $82 \% ; 95 \% \mathrm{CI}$, $66 \%-98 \% ; \mathrm{p}<0.0001$ ) (Table 2). At 6 weeks, the mean

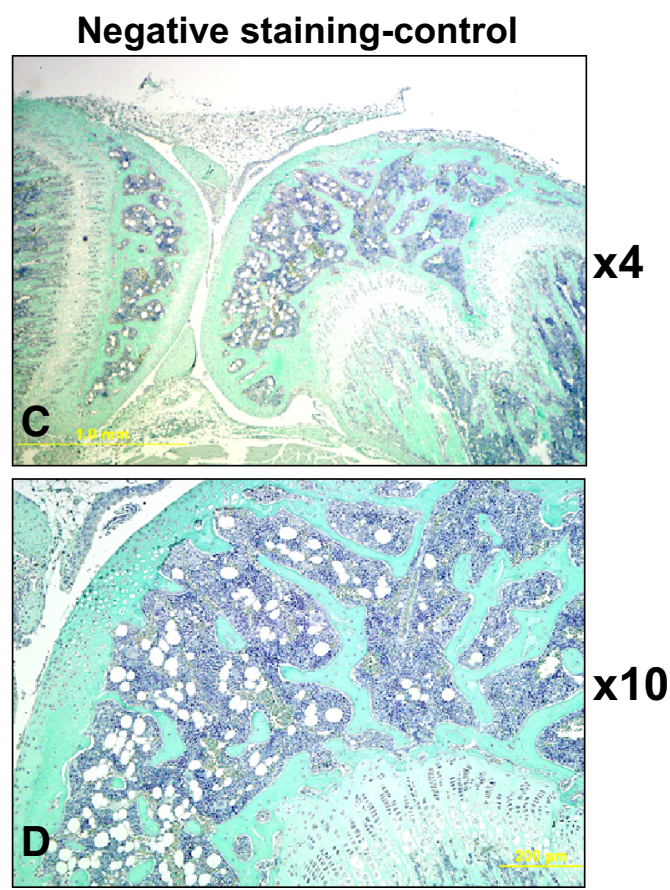

controls at $(\mathbf{C}) \times 4$ and $(\mathbf{D}) \times 10$ are shown for TUNEL staining method. There were five mice per group (Original magnification, $\times 4=1 \mathrm{~mm} ; \times 10=200 \mu$ ).

percent of TUNEL-positive osteocytes in the ischemic group decreased to $11 \% \pm 5 \%$ with no difference when compared with the control group ( $\mathrm{p}=0.56)$. 

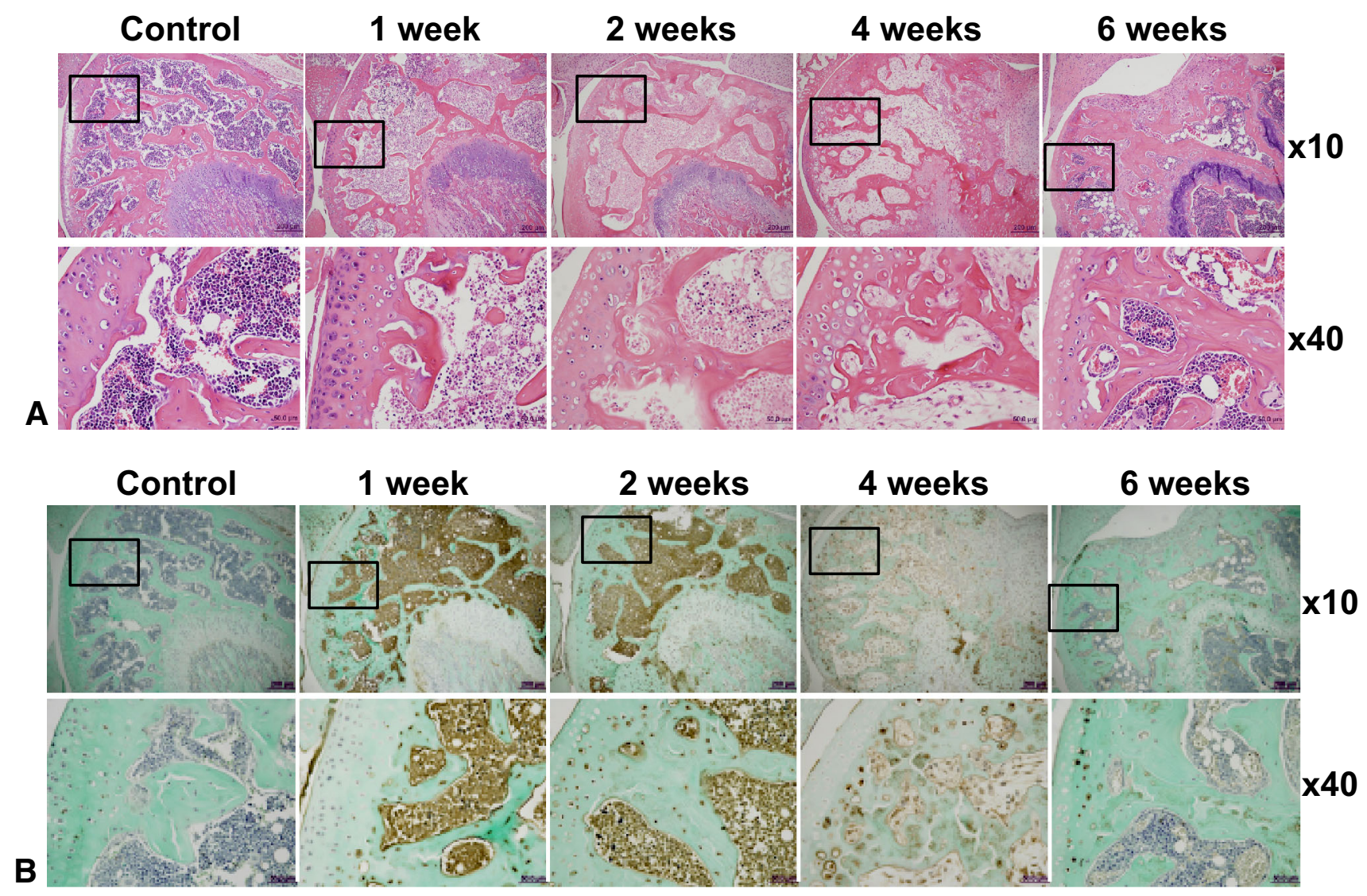

Fig. 5A-B Histologic analysis of the distal femur at 1, 2, 4, and 6 weeks after induction of ischemia. (A) At 1 week, extensive cell death was observed in the marrow space with loss of nuclear staining of the marrow cells, disorganized marrow structure, and necrotic blood vessels. By 2 weeks, vascular tissue invasion of the necrotic marrow space was observed with a progressive increase in infiltration of the necrotic marrow space with the vascular tissue at 4 and 6 weeks. At 6 weeks, some areas in the epiphysis had restoration of hematopoietic marrow. (Stain, hematoxylin and eosin; original magnification, $200 \mu=\times 40,50 \mu=\times 10$ ). (B) Diffuse TUNEL

The mean percent of empty lacunae increased from $1 \% \pm 1 \%$ in the control group to $43 \% \pm 17 \%$ in the ischemic group at 1 week (mean difference, $42 \% ; 95 \%$ CI, $22 \%-62 \% ; \mathrm{p}<0.0001$ ), $66 \% \pm 20 \%$ at 2 weeks (mean difference, 65\%; 95\% CI 44\%-85\%, p < 0.0001$)$, $78 \% \pm 15 \%$ at 4 weeks (mean difference, $77 \%$; $95 \% \mathrm{CI}$, $56 \%-97 \% ; \mathrm{p}<0.0001$ ) (Table 2). The mean percent of empty lacunae decreased to $38 \% \pm 8 \%$ at 6 weeks in comparison to the peak at the 4-week time but this value still was statistically significant compared with the value from the control group (mean difference, 36\%; 95\% CI, $16 \%-57 \% ; \mathrm{p}<0.0001$ ) (Fig. 6A).

The mean number of osteoblasts per bone surface $\left(/ \mu \mathrm{m}^{3}\right)$ was decreased in the ischemic group at 1 week (mean, $2.30 \pm 1.04 ; \mathrm{p}<0.0001)$ compared with the control group (mean, $31.07 \pm 3.51$ ) (Table 3). A gradual increase in the mean number of osteoblasts per bone surface was observed staining (brown) was observed at 1 and 2 weeks postischemia. There was substantial decrease in the TUNEL staining at 4 and 6 weeks owing to vascular tissue invasion of the marrow space and removal of the necrotic cell debris (Stain, TUNEL; original magnification, $200 \mu=\times 10 ; 50 \mu=\times 40$ ). The $\times 40$ images represent the rectangular area shown in $\times 10$ images. The control was the left distal femoral epiphysis at 1 week. There were eight mice per group for 1 , 2 , and 4 weeks and seven mice for 6 weeks. The $\times 40$ images in the lower panels represent the rectangular areas shown in the $\times 10$ images.

at 2 weeks (mean, $3.19 \pm 3.87 ; \mathrm{p}<0.0001$ ) and 4 weeks (mean, $11.19 \pm 8.34 ; \mathrm{p}<0.0001$ ) but the means were lower than the mean of the control group. At 6 weeks, the mean number of osteoblasts in the ischemic group (mean, $28.97 \pm 3.22$ ) also increased to a level similar to that of the control group with no difference between the two groups $(\mathrm{p}=0.91)$ (Fig. 6A).

The mean number of osteoclasts per bone surface $\left(/ \mu \mathrm{m}^{3}\right)$ was decreased in the ischemic group at 1 week (mean, $0.07 \pm 0.1 ; \mathrm{p}<0.001)$ compared with the number in the control group (mean, $4.88 \pm 1.99$ ) (Table 3 ). A gradual increase in the mean number of osteoclasts per bone surface was observed with a very low mean at 2 weeks (mean, $0.58 \pm 1.11 ; \mathrm{p}<0.0001)$ that increased from 2 weeks to 4 weeks (mean, $2.17 \pm 2.54 ; \mathrm{p}=0.02$ ) but the means were lower than the mean of the control group. At 6 weeks, the mean number of osteoclast in the ischemic group 
Table 2. Empty osteocyte lacunae, pyknotic osteocytes and TUNEL-positive osteocytes

\begin{tabular}{|c|c|c|c|c|c|}
\hline Parameter & $\begin{array}{l}\text { Control } \\
(\mathrm{n}=8)\end{array}$ & $\begin{array}{l}1 \text { week } \\
(\mathrm{n}=8)\end{array}$ & $\begin{array}{l}2 \text { weeks } \\
(\mathrm{n}=8)\end{array}$ & $\begin{array}{l}4 \text { weeks } \\
(\mathrm{n}=8)\end{array}$ & $\begin{array}{l}6 \text { weeks } \\
(\mathrm{n}=7)\end{array}$ \\
\hline $\begin{array}{l}\text { Empty lacunae }+ \text { lacunae with pyknotic osteocytes/total } \\
\text { number of lacunae }(\%)\end{array}$ & $1.3 \pm 1.2$ & $43.4 \pm 17.1^{*}$ & $66 \pm 19.8^{*}$ & $77.9 \pm 15.4^{*}$ & $37.7 \pm 7.9^{*}$ \\
\hline TUNEL + osteocytes/total number of osteocytes $(\%)$ & $2.2 \pm 1.1$ & $98.4 \pm 2.51 *$ & $92 \pm 6.5^{*}$ & $84.2 \pm 18.1 *$ & $10.5 \pm 10.5$ \\
\hline
\end{tabular}

TUNEL $=$ terminal deoxynucleotidyl transferase mediated dUTP nick-end labeling; ${ }^{*} \mathrm{p}<0.0001$ compared with control (unoperated left distal femoral epiphysis at 1 week); mean $\pm \mathrm{SD}$ are shown.
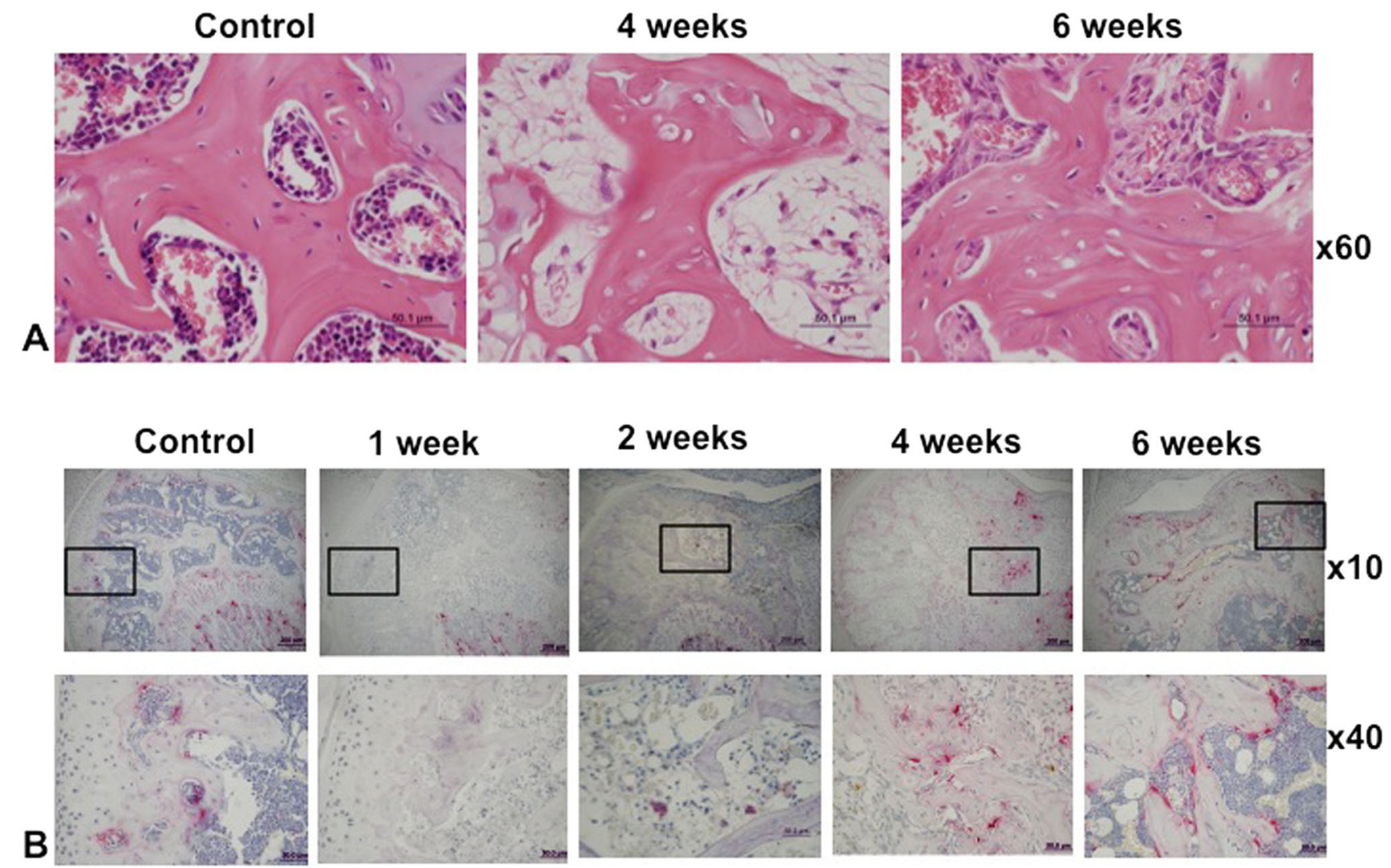

Fig. 6A-B Osteocyte lacunae, osteoblasts, and osteoclasts in the distal femur were evaluated from 1 to 6 weeks after the induction of ischemic osteonecrosis. Examples are shown of (A) normal bone with osteocytes in the lacunae (control), empty lacunae and absence of osteoblasts at 4 weeks postischemia, and appositional new bone with osteoblasts lining the bone surface and presence of osteocytes in the lacunae adjacent to the necrotic bone with empty lacunae 6 weeks

(mean, $3.01 \pm 1.57$ ) increased to a level similar to that of the control group with no difference between the two groups $(\mathrm{p}=0.23$; Fig. $6 \mathrm{~B})$.

\section{Morphologic Changes in the Model}

The mean bone volume percent was lower in the ischemic group compared with that of the control group from 2 to 6 weeks. The mean difference in the percent bone volume postischemia (Stain, hematoxylin and eosin; magnification, $\times 60$ ). (B) TRAP-stained sections showed a gradual increase in the number of osteoclasts (red) from 2 to 6 weeks postischemia. The $\times 40$ images in the lower panels represent the rectangular areas shown in the $\times 10$ images. There were eight mice per group for 1, 2, and 4 weeks and seven mice for 6 weeks (Original magnification, $\times 10=200 \mu ; \times 40$ and $\times 60=50 \mu)$.

(Fig. 7A) between the control and the ischemic group at 2, 4 , and 6 weeks was $5.5 \%$ (95\% CI, $0.9 \%-10.2 \%$; $\mathrm{p}=0.01), 5.3 \%(95 \% \mathrm{CI}, 0.6 \%-9.9 \% ; \mathrm{p}=0.02)$, and $6.0 \%$ (95\% CI, $1.1 \%-10.9 \% ; \mathrm{p}=0.008)$, respectively. The mean trabecular thickness (Fig. 7B) also was lower in the ischemic group compared with the control group from 2 to 6 weeks. The mean differences in trabecular thickness between the control and the ischemic group at 2, 4, and 6 weeks were $4.7 \mu \mathrm{m}^{3}$ (95\% CI, 1.3-8.2; $\left.\mathrm{p}=0.0006\right)$, $5.4 \mu \mathrm{m}^{3}$ (95\% CI, 2.0-8.9; $\left.\mathrm{p}<0.0001\right)$, and $4.5 \mu^{3}{ }^{3}(95 \%$ 
Table 3. Number of osteoclasts and osteoblasts in the distal femoral epiphysis after ischemic osteonecrosis

\begin{tabular}{lccccc}
\hline Parameter & Control $(\mathrm{n}=8)$ & 1 week $(\mathrm{n}=8)$ & 2 weeks $(\mathrm{n}=8)$ & 4 weeks $(\mathrm{n}=8)$ & 6 weeks $(\mathrm{n}=7)$ \\
\hline N.Oc/BS $\left(/ \mu \mathrm{m}^{3}\right)$ & $4.88 \pm 1.99$ & $0.07 \pm 0.1^{*}$ & $0.58 \pm 1.11^{*}$ & $2.17 \pm 2.54^{* *}$ & $3.01 \pm 1.57$ \\
N.Ob/BS $\left(/ \mu \mathrm{m}^{3}\right)$ & $31.07 \pm 3.51$ & $2.3 \pm 1.04^{*}$ & $3.19 \pm 3.87^{*}$ & $11.19 \pm 8.34^{*}$ & $28.97 \pm 3.22$ \\
\hline
\end{tabular}

$* \mathrm{p}<0.0001 ; * \mathrm{p}=0.02$ compared with control (unoperated left distal femoral epiphysis at 1 week); mean $\pm \mathrm{SD}$ are shown; $\mathrm{N} . \mathrm{Ob}=$ number of osteoblasts; N.Oc $=$ number of osteoclasts (TRAP + cells attached to bone); BS = bone surface.
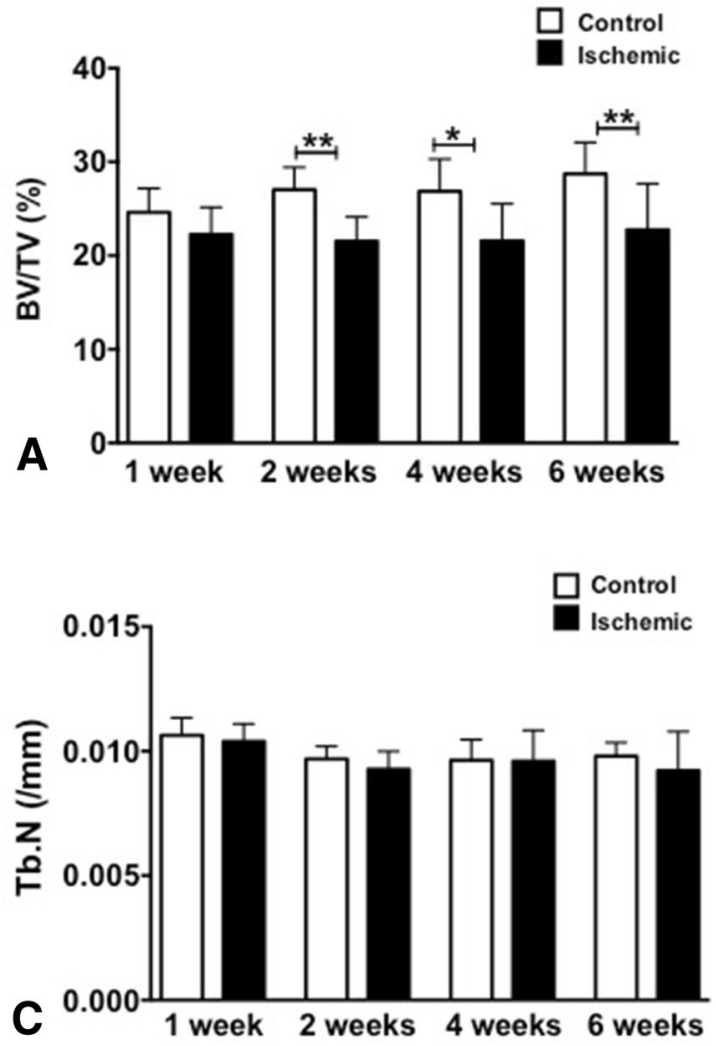

Fig. 7A-D (A) Bone volume percent (BV/TV\%), (B) trabecular thickness (Tb.Th), (C) trabecular number (Tb.N), and (D) trabecular separation (Tb.Sp) in the distal femoral epiphysis assessed by micro$\mathrm{CT}$ after induction of ischemic osteonecrosis are shown. The white

CI, 0.9-8.2; $\mathrm{p}=0.009$ ), respectively. No differences were found for the trabecular number and separation between the control and ischemic groups (Fig. 7C-D). No differences were found between the sham and the control groups (Table 4).

The shape of the distal femoral epiphysis was normal at 1 and 2 weeks postischemia. A mild deformity of the epiphysis was observed at 4 weeks, which became more severe at 6 weeks as assessed by measuring the height and diameter of the epiphysis using mid-coronal micro-CT images (Fig. 8A). At 6 weeks, the ratio of epiphyseal height to its diameter was lower in the ischemic group compared with the control group (control $0.74 \pm 0.03$ vs
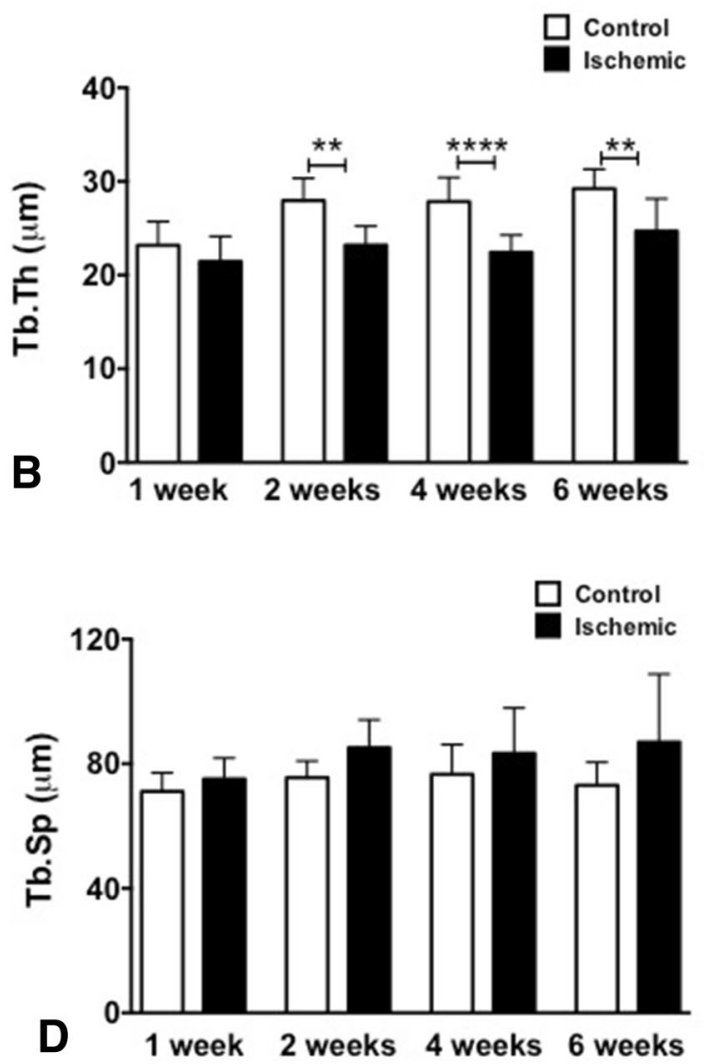

bars represent the normal left distal femoral epiphysis - the control, and the black bars represent the right distal femoral epiphysis - the ischemic side. There were 10 mice per group for 1,2 , and 4 weeks and nine mice for 6 weeks. ${ }^{*} \mathrm{p}<0.05 ; * * \mathrm{p}<0.01 ; * * * \mathrm{p}<0.0001$.

ischemic $0.66 \pm 0.03$; mean difference, $0.08 ; 95 \% \mathrm{CI}$, $0.00-0.16 ; \quad \mathrm{p}=0.03$ ) (Fig. 8B). Sham-operated mice showed no evidence of collapse.

\section{Discussion}

Availability of a reliable mouse model of ischemic osteonecrosis could accelerate our understanding of the molecular pathways involved in the repair process after ischemic osteonecrosis and the development of novel therapeutic strategies to stimulate bone healing; however, no mouse model of ischemic osteonecrosis is currently 
Table 4. Micro-CT analysis of the distal femoral epiphysis after sham operation

\begin{tabular}{|c|c|c|c|c|c|c|c|c|}
\hline \multirow[t]{2}{*}{ Parameter } & \multicolumn{2}{|c|}{1 week $(\mathrm{n}=5)$} & \multicolumn{2}{|c|}{2 weeks $(\mathrm{n}=5)$} & \multicolumn{2}{|c|}{4 weeks $(n=5)$} & \multicolumn{2}{|c|}{6 weeks $(\mathrm{n}=5)$} \\
\hline & Control & Sham & Control & Sham & Control & Sham & Control & Sham \\
\hline BV/TV (\%) & $24.8 \pm 1.3$ & $25.1 \pm 2.4$ & $19.8 \pm 1.2$ & $20.5 \pm 1.5$ & $19.3 \pm 2.8$ & $20.7 \pm 0.5$ & $29.5 \pm 1.9$ & $30.3 \pm 2.6$ \\
\hline Tb.Th $(\mu \mathrm{m})$ & $24.4 \pm 0.7$ & $24.9 \pm 1.9$ & $25.3 \pm 0.6$ & $25.3 \pm 1$ & $26.7 \pm 1.6$ & $26.7 \pm 0.8$ & $29.6 \pm 2.4$ & $29.1 \pm 1.3$ \\
\hline Tb.N (/mm) & $10.2 \pm 0.4$ & $10.1 \pm 0.2$ & $7.8 \pm 0.4$ & $8.1 \pm 0.5$ & $7.2 \pm 0.7$ & $7.8 \pm 0.3$ & $10 \pm 0.5$ & $10.4 \pm 0.6$ \\
\hline Tb.Th $(\mu \mathrm{m})$ & $74.2 \pm 3.8$ & $74.9 \pm 3.5$ & $102.9 \pm 15.1$ & $98.3 \pm 7.3$ & $112.9 \pm 15.1$ & $101.7 \pm 4.8$ & $70.8 \pm 3.9$ & $67.4 \pm 6.3$ \\
\hline
\end{tabular}

No statistically significant differences were detected between the control and sham groups; mean \pm SD values are shown; BV/TV $=$ bone volume/tissue volume; $\mathrm{Tb} . \mathrm{Th}=$ trabecular bone thickness; $\mathrm{Tb} . \mathrm{N}=$ trabecular bone number; $\mathrm{Tb} . \mathrm{Sp}=$ trabecular bone separation.
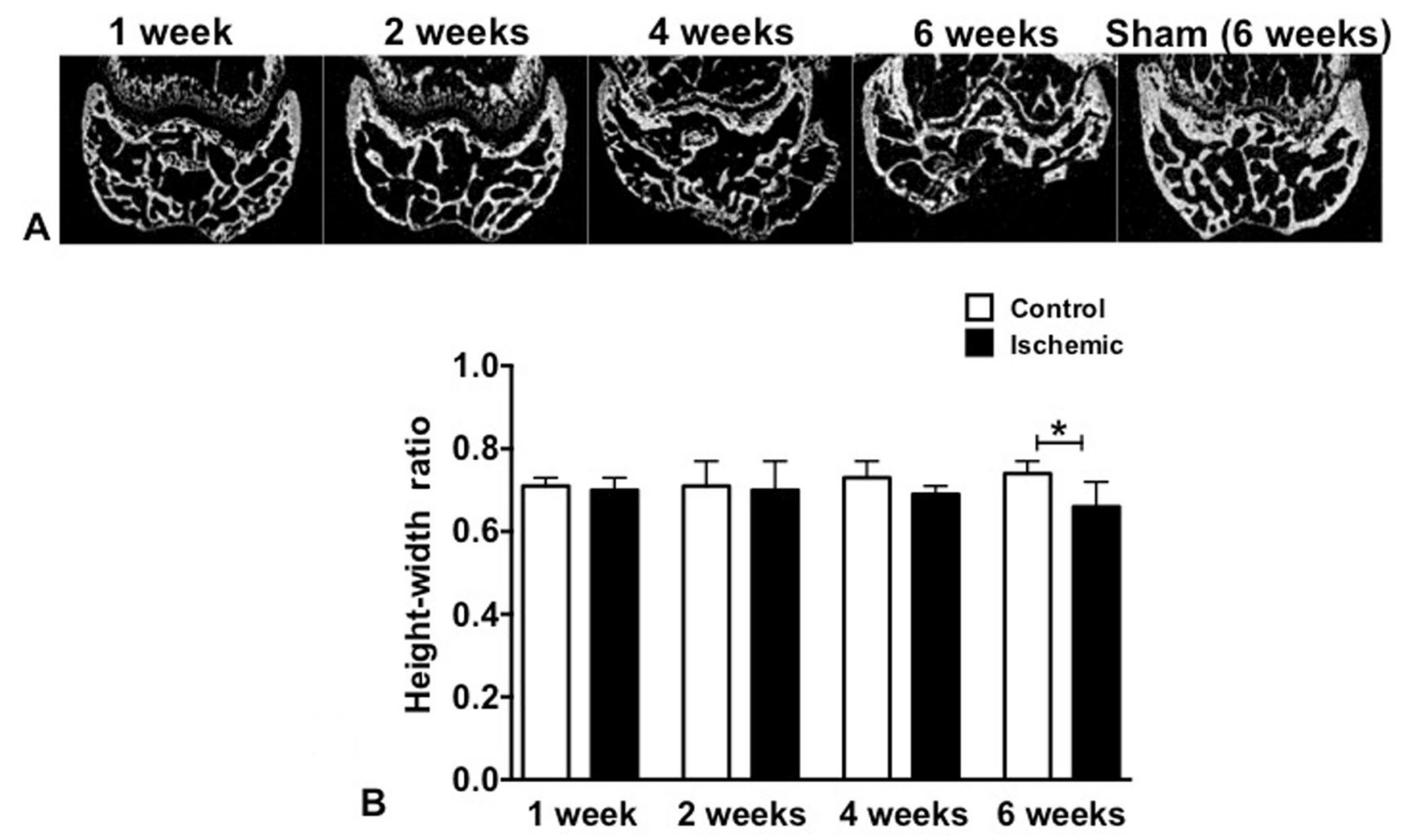

Fig. 8A-B (A) Representative mid-coronal micro-CT images of the distal femoral epiphysis are shown. A deformity of the epiphysis was present at 6 weeks postischemia. There were 10 mice per group at 1 , 2, and 4 weeks and nine mice at 6 weeks. (B) A bar graph shows the

available. We developed a reliable mouse model of ischemic osteonecrosis of the distal femoral epiphysis using a systematic approach to define the vascular anatomy of the distal femur followed by performing selective disruption of the blood vessels supplying the bone using microsurgical techniques. To our knowledge, this is the first surgical model of ischemic osteonecrosis in mice. We found that cauterization of all four vessels supplying the distal femoral epiphysis was required to induce an extensive ischemic osteonecrosis in the epiphysis. All 15 animals had extensive osteonecrosis develop in the distal femoral ratio of the distal femoral epiphyseal height to its width, as determined using mid-coronal micro-CT images. $* \mathrm{p}<0.05$, control versus ischemic side.

epiphysis after four-vessel cauterization, indicating that the model is reliable in terms of inducing severe osteonecrosis.

The study has limitations. Histologic assessment of revascularization using specific immunostaining for the presence of endothelial cells, such as CD 31 or platelet endothelial cell adhesion molecule (PECAM), was not performed. Histomorphometric assessment of trabecular bone parameters was not performed. The results of this study are applicable for ischemic osteonecrosis involving a growing bone as relatively young mice were used owing to 
our interest in studying ischemic osteonecrosis affecting children. In the pediatric population, ischemic osteonecrosis is seen in various conditions including trauma, dislocation, iatrogenic injuries related to treatment of hip dysplasia, and Legg-Calvé-Perthes disease [22, 24]. Given the findings related to osteonecrosis in young mice, it would be interesting to investigate how the age of the animal affects bone healing after ischemic osteonecrosis using the mouse model.

The first question we asked in this study was whether we can generate a mouse model of osteonecrosis by cauterizing the blood supply to the distal femoral epiphysis. As a preamble to this discussion, we selected the distal femoral epiphysis as the target bone instead of the proximal femoral epiphysis as it is one of the largest bone segments found in mice. It is approximately four times larger than the proximal femoral epiphysis, providing a greater amount of tissue for research and analyses [18]. The more superficial location of the distal femoral epiphysis compared with the proximal femoral epiphysis also made this site preferable as it technically is easier to induce ischemic osteonecrosis using microsurgery with less risk of complications such as hip dislocation owing to extensive soft tissue dissection. The need to cauterize all four vessels to obtain total epiphyseal osteonecrosis in this model is not surprising given that the knee region is well vascularized and has collateral circulation which can compensate for one or more vessel disruptions. In terms of a mouse model of osteonecrosis, we are aware of only one other model-a mouse model of glucocorticoid-induced osteonecrosis reported by Yang et al. [47]. The pathogenesis of glucocorticoid-induced osteonecrosis is very different from that of ischemic osteonecrosis, such that the two models have different implications and utility in terms of investigating the pathophysiology of two distinct etiologies of osteonecrosis. We believe that the availability of both mouse models (ischemia-induced and glucocorticoid-induced osteonecrosis) will be useful in advancing our understanding of osteonecrosis. The incidence of glucocorticoid-induced osteonecrosis in BALB/cJ mice in which dexamethasone was systematically administered was less than 50\%. In addition, the location of the osteonecrosis was sporadic. In contrast, our surgically induced ischemic osteonecrosis model showed a $100 \%$ incidence of severe osteonecrosis specifically in the distal femoral epiphysis.

The second question we asked was what are the histologic changes in the model? Histologic assessment showed diffuse cell death in the epiphysis as seen with TUNEL staining results. The TUNEL staining was sensitive in showing extensive cell death from an early time. At 1 week postischemia, $96 \%$ of the osteocytes were found to be TUNEL positive. Quantitation of empty lacunae further confirmed the diffuse osteocyte death in the trabecular bone but this sign of osteonecrosis took more time to fully manifest with the highest percent of empty lacunae being observed 4 weeks postischemia. The diffuse cell death was followed by initiation of revascularization of the necrotic marrow space from the periphery of the epiphysis at 2 weeks. By 6 weeks, necrotic marrow space was completely replaced by fibrovascular tissue and in some areas by hematopoietic marrow. The histologic characteristics of osteonecrosis in our mouse model are consistent with those described in human patients and in other models of vascular disruption-induced ischemic osteonecrosis [4, 5, 49] which feature extensive cell death (osteoblasts, osteocytes, and marrow cells) and empty lacunae. In comparison to the rat model of osteonecrosis [14, 29, 38], our mouse model showed similar histologic changes and the temporal sequence of the repair process. Revascularization of the necrotic marrow space was followed by a gradual increase in the number of osteoclasts and osteoblasts on the bone surfaces from 2 to 6 weeks. Furthermore, it appears to have a relatively fast healing process as seen in the rat model $[14,29,38]$ compared with that of large animal models of osteonecrosis [25, 26] and human disease [35]. The fast repair also may be related to relatively young mice (5 weeks old) used in this study. The sequential changes following ischemic induction were cell death, revascularization, resorption, and new bone formation (Fig. 9).

The third question we asked regarded morphologic changes in the model. In this study, micro-CT was used to assess the morphologic features of the trabecula. This automated imaging technique allowed quantitative assessment of the whole epiphysis and assessment of the overall
Fig. 9 The schema represents the histologic observations regarding the timing of cell death, revascularization, resorption, and new bone formation in our mouse model of ischemic necrosis.

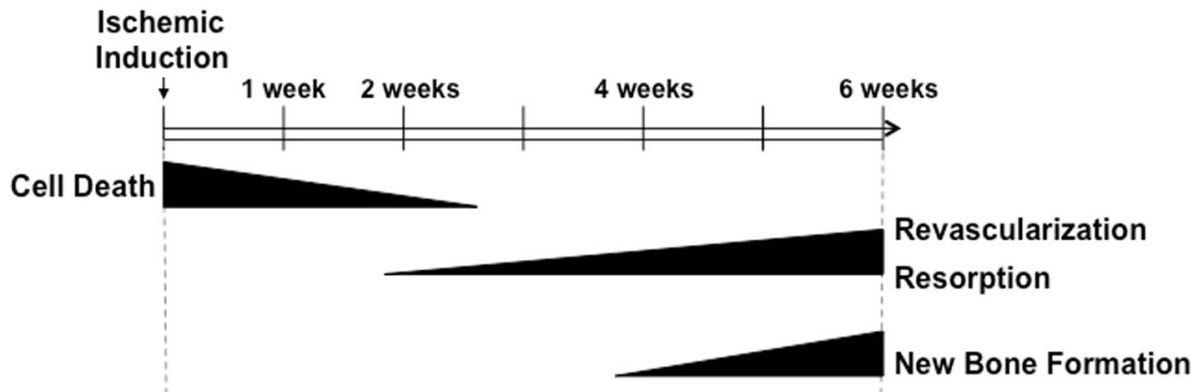


shape of the epiphysis. This model however, had an epiphyseal deformity develop, as measured by the ratio of epiphyseal height to its diameter, which was statistically significant at 6 weeks. This may be an important feature of the model as some animal models of osteonecrosis do not have collapse or deformity of the epiphysis develop. Development of bone deformity, which can lead to the early onset of osteoarthritis, is seen in patients and in some large animal models of ischemic osteonecrosis $[7,22,25$, $33,35]$. This feature also may be used to evaluate whether a potential treatment for osteonecrosis can prevent the development of the deformity. A comparison of the mean bone volume percent in the control and the ischemic sides revealed a decrease of the mean in the ischemic side by $5 \%$ to $6 \%$, which was statistically significant at 2,4 , and 6 weeks. The mean trabecular thickness also was significantly decreased during these times, with the mean difference between the control and ischemic sides ranging from 4 to $5 \mu \mathrm{m}$. Since micro-CT provides only static measurements of the trabecular bone, it is not clear if the decrease in the bone volume and trabecular thickness on the ischemic side are attributable to an imbalance of bone resorption and bone formation. As noted above, we observed a gradual increase in the number of osteoclasts and osteoblasts on bone surfaces from 2 to 6 weeks postischemia. Taken together, these results suggest that remodeling of the necrotic bone is imbalanced by a relative increase in bone resorption and relative decrease in new bone formation which produce a net bone loss. We did not perform calcein labeling to determine the mineral apposition and bone formation rates. This is another limitation of this study in which dynamic parameters of bone formation were not assessed.

We developed a new mouse model of ischemic osteonecrosis that produced extensive cell death in the distal femoral epiphysis which developed a deformity with time. We believe that this mouse model may be a useful tool to study potential therapeutic strategies to improve bone healing after ischemic osteonecrosis.

Acknowledgments We thank ChanHee Jo $\mathrm{PhD}$ for statistical analysis, Amanda McLerran LVT, RVLAT for animal care and surgical assistance, Reuel Cornelia BSMT, AMT and Richard Banlaygas BSMT, AMT for histologic preparation, and Ila Oxendine MS for technical assistance. All are affiliated with the Center of Excellence in Hip Disorders, Texas Scottish Rite Hospital for Children, Dallas, TX, USA.

\section{References}

1. Assouline-Dayan Y, Chang C, Greenspan A, Shoenfeld Y, Gershwin ME. Pathogenesis and natural history of osteonecrosis. Semin Arthritis Rheum. 2002;32:94-124.

2. Boss JH, Misselevich I. Experimental avascular osteonecrosis. Current Orthopaedics. 2001;15:57-67.
3. Boss JH, Misselevich I. Osteonecrosis of the femoral head of laboratory animals: the lessons learned from a comparative study of osteonecrosis in man and experimental animals. Vet Pathol. 2003;40:345-354.

4. Calder JD, Buttery L, Revell PA, Pearse M, Polak JM. Apoptosis: a significant cause of bone cell death in osteonecrosis of the femoral head. J Bone Joint Surg Br. 2004;86:1209-1213.

5. Calvert PT, Kernohan JG, Sayers DC, Catterall A. Effects of vascular occlusion on the femoral head in growing rabbits. Acta Orthop Scand. 1984;55:526-530.

6. Chen Y, Whetstone HC, Lin AC, Nadesan P, Wei Q, Poon R, Alman BA. Beta-catenin signaling plays a disparate role in different phases of fracture repair: implications for therapy to improve bone healing. PLoS Med. 2007;4:e249.

7. Conzemius MG, Brown TD, Zhang Y, Robinson RA. A new animal model of femoral head osteonecrosis: one that progresses to human-like mechanical failure. J Orthop Res. 2002;20:303-309.

8. Drescher W, Beckmann R, Kasch R, Pufe M, Knobe M, Kweider N, Hassenpflug J, Tingart M, Pufe T, Kadyrov M. Nitrate patch prevents steroid-related bone necrosis. J Orthop Res. 2011;29:1517-1520.

9. Duvall CL, Taylor WR, Weiss D, Guldberg RE. Quantitative microcomputed tomography analysis of collateral vessel development after ischemic injury. Am J Physiol Heart Circ Physiol. 2004;287:H302-310.

10. Einhorn TA, Gerstenfeld LC. Fracture healing: mechanisms and interventions. Nat Rev Rheumatol. 2015;11:45-54.

11. Fan M, Peng J, Qin L, Lu S. Experimental animal models of osteonecrosis. Rheumatol Int. 2011;31:983-994.

12. Gerstenfeld LC, Cho TJ, Kon T, Aizawa T, Tsay A, Fitch J, Barnes GL, Graves DT, Einhorn TA. Impaired fracture healing in the absence of TNF-alpha signaling: the role of TNF-alpha in endochondral cartilage resorption. J Bone Miner Res. 2003;18: 1584-1592.

13. Heilmann A, Schinke, T, Bindl R, Wehner T, Rapp A, HaffnerLuntzer M, Neimitz C, Liedert A, Amling M, Ignatius A. The Wnt serpentine receptor Frizzled-9 regulates new bone formation in fracture healing. PLoS One. 2013;8:e84232.

14. Hirano T, Iwasaki K, Yamane Y. Osteonecrosis of the femoral head of growing, spontaneously hypertensive rats. Acta Orthop Scand. 1988;59:530-535.

15. Hofstaetter JG, Wang J, Yan J, Glimcher MJ. Changes in bone microarchitecture and bone mineral density following experimental osteonecrosis of the hip in rabbits. Cells Tissues Organs. 2006;184:138-147.

16. Irisa $\mathrm{T}$, Yamamoto $\mathrm{T}$, Miyanishi $\mathrm{K}$, Yamashita A, Iwamoto $\mathrm{Y}$, Sugioka Y, Sueishi K. Osteonecrosis induced by a single administration of low-dose lipopolysaccharide in rabbits. Bone. 2001;28:641-649.

17. Iwakiri K, Oda Y, Kaneshiro Y, Iwaki H, Masada T, Kobayashi A, Asada A, Takaoka K. Effect of simvastatin on steroid-induced osteonecrosis evidenced by the serum lipid level and hepatic cytochrome P4503A in a rabbit model. J Orthop Sci. 2008; 13:463-468.

18. Jerome C, Hoch B. Skeletal system. In: Treuting PM, Dintzis S, eds. Comparative Anatomy and Histology: A Mouse and Human Atlas. Oxford, UK: Elsevier; 2012:53-70.

19. Kabata T, Kubo, T, Matsumoto T, Hirata, T, Fujioka M, Takahashi KA, Yogishita S, Kabayashi M, Tomita K. Onset of steroidinduced osteonecrosis in rabbits and its relationship to hyperlipaemia and increased free fatty acids. Rheumatology (Oxford). 2005;44:1233-1237.

20. Keijser LC, Schreuder HW, Buma P, Weinans H, Veth RP. Cryosurgery in long bones: an experimental study of necrosis and revitalization in rabbits. Arch Orthop Trauma Surg. 1999;119:440-444. 
21. Kenzora JE, Steele RE, Yosipovitch ZH, Glimcher, MJ. Experimental osteonecrosis of the femoral head in adult rabbits. Clin Orthop Relat Res. 1978;130:8-46.

22. Kim HK. Pathophysiology and new strategies for the treatment of Legg-Calve-Perthes disease. J Bone Joint Surg Am. 2012;94:659-669.

23. Kim HK, Bian H, Randall T, Garces A, Gerstenfeld LC, Einhorn TA. Increased VEGF expression in the epiphyseal cartilage after ischemic necrosis of the capital femoral epiphysis. J Bone Miner Res. 2004;19:2041-2048.

24. Kim HK, Larson AN, Fletcher ND, Winick N, Kim Y. Childhood femoral head osteonecrosis. Clin Rev Bone Miner Metab. 2011;9:2-12.

25. Kim HK, Su PH. Development of flattening and apparent fragmentation following ischemic necrosis of the capital femoral epiphysis in a piglet model. J Bone Joint Surg Am. 2002;84: 1329-1334.

26. Kim HK, Su PH, Qiu YS. Histopathologic changes in growthplate cartilage following ischemic necrosis of the capital femoral epiphysis: an experimental investigation in immature pigs. $J$ Bone Joint Surg Am. 2001;83:688-697.

27. Komatsu DE, Mary MN, Schroeder RJ, Robling AG, Turner CH, Warden SJ. Modulation of Wnt signaling influences fracture repair. J Orthop Res. 2010;28: 928-936.

28. Kothapalli R, Aya-ay JP, Bian H, Garces A, Kim HK. Ischaemic injury to femoral head induces apoptotic and oncotic cell death. Pathology. 2007;39:241-246.

29. Levin D, Norman D, Zinman C, Rubinstein L, Sabo E, Misselevich I, Reis D, Boss JH. Treatment of experimental avascular necrosis of the femoral head with hyperbaric oxygen in rats: histological evaluation of the femoral heads during the early phase of the reparative process. Exp Mol Pathol. 1999;67:99-108.

30. Loizou CL, Parker MJ. Avascular necrosis after internal fixation of intracapsular hip fractures: a study of the outcome for 1023 patients. Injury. 2009;40:1143-1146.

31. Malizos KN, Quarles LD, Seaber AV, Rizk WS, Urbaniak JR. An experimental canine model of osteonecrosis: characterization of the repair process. J Orthop Res. 1993;11:350-357.

32. Matsui M, Ohzono K, Nakamura N, Sugano N, Masuhara K, Nakata, K, Takaoka K, Ono K, Ochi T. The immune reaction to heterologous serum causes osteonecrosis in rabbits. Virchows Arch. 1995;427:205-211.

33. McAndrew MP, Weinstein SL. A long-term follow-up of LeggCalve-Perthes disease. J Bone Joint Surg Am. 1984;66: 860-869.

34. Mickelson MR, McCurnin DM, Awbrey BJ, Maynard JA, Martin RK. Legg-Calve-Perthes disease in dogs: a comparison to human Legg-Calve-Perthes disease. Clin Orthop Relat Res. 1981; 157:287-300
35. Mont MA, Jones LC, Hungerford DS. Nontraumatic osteonecrosis of the femoral head: ten years later. J Bone Joint Surg Am. 2006;88:1117-1132.

36. Nakata K, Masuhara, K, Nakamura, N, Shibuya T, Sugano N, Matsui M, Ochi T, Ohzono, K. Inducible osteonecrosis in a rabbit serum sickness model: deposition of immune complexes in bone marrow. Bone. 1996;18:609-615.

37. Nishino M, Matsumoto T, Nakamura T, Tomita K. Pathological and hemodynamic study in a new model of femoral head necrosis following traumatic dislocation. Arch Orthop Trauma Surg. 1997;116:259-262.

38. Norman D, Reis DN, Zinman C, Misselevich I, Boss JH. Vascular deprivation-induced necrosis of the femoral head of the rat: an experimental model of avascular osteonecrosis in the skeletally immature individual or Legg-Perthes disease. Int J Exp Pathol. 1998;79:173-181.

39. Rammelt S, Zwipp H. Talar neck and body fractures. Injury. 2009;40:120-135.

40. Reed KL, Brown TD, Conzemius MG. Focal cryogen insults for inducing segmental osteonecrosis: computational and experimental assessments of thermal fields. J Biomech. 2003;36:1317-1326.

41. Seiler JG 3rd, Kregor PJ, Conrad EU 3rd, Swiontkowski MF. Posttraumatic osteonecrosis in a swine model: correlation of blood cell flux, MRI and histology. Acta Orthop Scand.1996;67: 249-254.

42. Szabo RM, Manske D. Displaced fractures of the scaphoid. Clin Orthop Relat Res. 1988;230:30-38.

43. Tsuji K, Bandyopadhyay A, Harfe B D, Cox K, Kakar S, Gerstenfeld L, Einhorn T, Tabin CJ, Rosen V. BMP2 activity, although dispensable for bone formation, is required for the initiation of fracture healing. Nat Genet. 2006;38:1424-1429.

44. Velez R, Soldado F, Hernandez A, Barber I, Aguirre M. A new preclinical femoral head osteonecrosis model in sheep. Arch Orthop Trauma Surg. 2011;131:5-9.

45. Wang Y, Yin L, Li Y, Liu P, Cui Q. Preventive effects of puerarin on alcohol-induced osteonecrosis. Clin Orthop Relat Res. 2008;466:1059-1067.

46. Weinstein RS. Glucocorticoid-induced osteonecrosis. Endocrine. 2012;41:183-190.

47. Yang L, Boyd K, Kaste SC, Kamdem Kamdem L, Rahija RJ, Relling MV. A mouse model for glucocorticoid-induced osteonecrosis: effect of a steroid holiday. J Orthop Res. 2009;27: 169-175.

48. Yeranosian M, Horneff, JG, Baldwin K, Hosalkar HS. Factors affecting the outcome of fractures of the femoral neck in children and adolescents: a systematic review. Bone Joint J. 2013;95:135-142.

49. Youm YS, Lee SY, Lee SH. Apoptosis in the osteonecrosis of the femoral head. Clin Orthop Surg. 2010;2:250-255. 